\title{
The Argos System: MONITORING THE WORLD's ENVIRONMENT
}

\author{
By Katherine R. Swenson and A. E. Shaw III
}

$T_{H}$ HE ARGOS SYSTEM is a satellitebased Data Collection and Location System (DCLS) used to collect in situ environmental data from fixed and mobile platforms. Coupled with an accurate position determination, these data are time-tagged and disseminated world-wide. The year 1990 marks twelve years of continuous operation of the Argos system, primarily in the service of oceanography.

Argos is the result of a cooperative venture between the Centre National d'Etudes Spatiales, (CNES-the French space agency), the National Aeronautic and Space Administration (NASA), and the National Oceanic and Atmospheric Administration (NOAA). A Memorandum of Understanding (MOU) was signed in 1974 defining each partner's responsibilities. The system became operational in October of 1978 with the launch of the protoflight satellite, TIROS-N. An updated bilateral agreement, signed in March 1986, insures continued joint cooperation through the rest of the TIROS series (into the late1990s). CNES is planning future joint space cooperation with NOAA involving possible additions to the NOAA/TIROS-N series and also succeeding-generation polar orbiting space platforms.

The Argos system is reserved for programs involved with the collection of environmental data; defined as measurements of physical, chemical or biological properties of solid earth, earth's waters (rivers, lakes and oceans), and the atmosphere (including space). Programs not directly involved with environmental monitoring may be approved by the Operations Committee on a short-term basis. A bilateral France-USA Operations Committee is re-

Katherine R. Swenson and A. E. Shaw III, Service Argos, Inc., 1801 McCormick Drive, Suite 10, Landover, MD 20785.

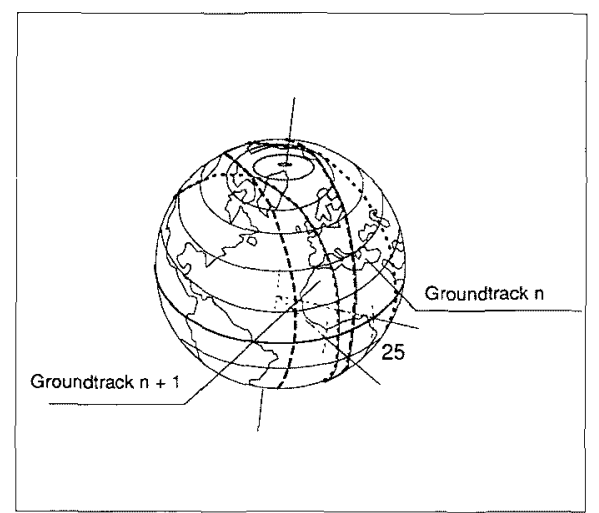

Fig. 1: Argos swath overlap

sponsible for ensuring that the system is managed according to the MOU.

The Argos DCLS comprises four essential components: platforms equipped with Platform Transmitter Terminals, satellites, receiving stations, and the Global Processing Centers.

\section{Platform Transmitter Terminals}

An Argos-certified Platform Transmitter Terminal (PTT) is a data acquisition station designed for transmission via the Argos system. Argos PTTs are simple to operate, light $(<1 \mathrm{~kg})$, inexpensive, and use little power ( $200 \mathrm{~mW}$ on average). PTTs can be mounted on many types of terrestrial and maritime platforms; such as drifting buoys, balloons, animals, ships, trucks or land-based stations.

Since Argos is a random access system, the PTTs uplink (transmit) their messages at preset intervals without satellite interrogation. Each message may contain up to 256 bits of sensor data. A message uplink takes between 360 and $920 \mathrm{~ms}$, as a function of message length. The uplink timeout, or repetition period, is normally set between 50 and 200 seconds, depending on the user's application. All PTTs transmit at 401.65 MHz. Separation in time is achieved through asynchronization of the transmission and by use of different repetition periods. PTT location is determined by measuring the Doppler shift of the carrier frequency transmitted by each PTT. The probability of collecting data from a PTT during a given spacecraft pass is $99 \%$, providing the messages are identical during the whole overpass (approximately 10 minutes).

\section{The Satellites}

The Argos onboard package is carried by two spacecraft (currently NOAA-10 and NOAA-11) simultaneously in circular, nearpolar, sun-synchronous orbits to provide the Argos system with complete global coverage. Sun-synchronous means that each satellite orbit transects the equatorial plane at fixed local solar times. Orbit revolution period is approximately 101 minutes.

The instantaneous field of view of each satellite is a circular footprint approximately $5000 \mathrm{~km}$ in diameter. The satellites receive messages from the PTTs within the footprint, time-codes them, measures the frequency, and demodulates the PTT ID number and sensor data. Four messages can be received and processed simultaneously. With the launch of NOAA-K in 1993, and subsequent spacecraft, an additional four Data Record Units will be added, increasing the capacity of the Argos system fourfold.

The swath created by the satellite's footprint as it sweeps the globe is displaced $25^{\circ}$ in longitude to the west, at the equator, every satellite revolution, due to rotation of the earth. There is an element of swath overlap for each successive orbit (Fig. 1). Global coverage was guaranteed by selection of this type of orbital geometry. The number of passes over a given location each day is a function of latitude. Satellite coverage ranges from six passes per day at the equator to twenty-eight passes per day at the poles. 
Argos data are "played back" from the satellites in three modes:

- The S-band which transmits the recorded data on $1698.0,1702.5$, or 1707.0 $\mathrm{MHz}$ at 2.66 mbps.

- The real-time S-band on 1698.0 or $1707.0 \mathrm{MHz}$ at $665.4 \mathrm{kbps}$.

- The real-time VHF beacon on 136.77 or $137.77 \mathrm{MHz}$ at $8.32 \mathrm{kbps}$.

\section{Telemetry Receiving Stations}

Argos currently utilizes three major telemetry receiving stations (Wallops Island, VA, and Fairbanks, AK, in the United States, and Lannion in France) where all the recorded data are downlinked when a satellite is within station visibility.

All Argos data received from the U.S. stations are routed through NOAA's National Environmental Satellite, Data and Information Service facility in Suitland, Maryland, for decommutation. These data are then transmitted to the two Global Processing Centers (GPCs).

Until mid-87, Service Argos received and processed only the recorded data stored onboard the satellite and played back approximately ten times per day per satellite. With the satellite orbital period being 101 minutes, processing delays from the time of PTT data transmission to on-line data availability were usually about two hours for the recorded data. However, since July 1987 , Argos has received the processed DCLS data (imbedded in NOAA's High-Resolution Pixel Transmission data stream) in near real-time. Today, ten real-time passes are acquired by Service Argos.

Service Argos is currently investigating the possibility of receiving data through another receiving station in the western US to reduce the data throughput times and provide real-time coverage to all of North America.

\section{Global Processing Centers (GPCs)}

The Argos system is administered by Service Argos, Inc., in North America, and by Collecte Localisation Satellite (CLS)/ Service Argos, S.A., in Toulouse, France. Redundant, identical processing centers are in place at each of these facilities, where the following operations are performed:

- Decoding of the received PTT messages and conversion of data into physical units, as specified by the end user,

- Very accurate computation of the satellite(s) orbits,

- PTT location computation using orbital data and the Doppler frequency shifts,

- Storage of all these results in computer files for on-line user access,

- Generation of the archived data base for each program and PTT.

Each of the processing centers is able to take on the entire global processing load should one of the GPCs fail.

\section{Location Calculation and Quality}

PTT location is determined by measuring the Doppler shift of the carrier frequency (401.65 MHz) of messages received by the spacecraft. Since the transmission frequency is fixed for all PTTs, the satellite-received frequency at any instant can be used to define the field of possible positions for a PTT. The field is in the form of a half-cone, with the satellite at its apex, the satellite velocity vector as the axis of symmetry, and the apex half-angle (A) such that:

$$
\cos (A)=\frac{\left(f_{r}-f_{e}\right)}{f_{e}} * \frac{c}{V}
$$

Where:

$c=$ Speed of light

$\mathrm{V}=$ Satellite speed relative to PTT

$\mathrm{f}_{\mathrm{e}}=$ Transmission frequency $(401.65$ $\mathrm{MHz}$ )

$\mathrm{f}_{\mathrm{r}}=$ Receive frequency

In April 1987, three different levels of location accuracy were implemented (Class 1.2 and 3). Since January 1988, a new class (Class 0 ) has been defined to accommodate the needs of certain users. The quality index depends on location quality control and geometric conditions. The user may choose between these four classes of location accuracy described below:

- Class 0-Special Location: Service based on a minimum of two messages received by the satellite. Both Doppler location solutions are given; therefore, the quality of the result is left to the user's determination.

- Class 1-Non-Guaranteed Location: (Accuracy: $68 \%$ of the results are within 1 $\mathrm{km}$ of the true position.) Based on at least four messages being received by the satellite, with an interval of at least 240 seconds between the first and last message received. The location is not necessarily of low quality, since it may be a location for which it was not possible to conduct all of the quality control checks.

- Class 2-Standard Location: (Accuracy: $68 \%$ of the results within $350 \mathrm{~m}$ of the true position.) At least five messages received over a duration of at least $420 \mathrm{sec}$ onds. Requires good short-term oscillator stability. There is no ambiguity in the solution.
- Class 3-Quality Location: (Accuracy: $68 \%$ of the results within $150 \mathrm{~m}$ of the true position.) Uses the same criteria as that for standard location, plus favorable geometric conditions of the PTT relative to the space-craft ground track and oscillator stability providing good internal consistency. Data Distribution

Processed data are available generally within three hours after the receipt of data from the satellite(s) in computer files accessible by the user through telephone, telex or telecommunication networks (i.e., TYMNET or TRANSPAC). These networks may be inter-connected through international transit nodes.

There are three types of on-line files that contain processed data:

- The AJOUR file containing the most recent location and sensor message for each PTT.

- The TELEX file containing in chronological order the most significant sensor message for each PTT and the corresponding location for each satellite pass received during the past 100 hours.

- The DISPOSE file containing all location data and all sensor messages for each satellite pass and each PTT received during the past 100 hours in chronological order.

A Direct Data Distribution service is also available as an alternative to dial-up for users who require their data immediately following processing or at set intervals during the day (not to exceed four times per day). It is possible to request that only real-time data, only recorded data, or both real-time and recorded data be sent to your site. The Direct Data Distribution service is also available to users who have access to computer networks such as SPAN, since the interconnection protocol is the same whether the receiving system is a computer or a network.

Data are also available on computer compatible tapes, floppy disks or printouts on a fortnightly or monthly basis. Data are archived for three months. Appropriately formatted data may be distributed over the Global Telecommunication System.

\section{Applications for Argos}

Argos PTTs typically transmit oceanographic and atmospheric parameters: atmospheric pressure, air temperature, seasurface temperature and temperature at various ocean depths, wind speed and direction, and currents. Argos applications include weather monitoring from various types of vessels, guidance of fishing fleets, iceberg drift prediction, hydrological monitoring, and tracking of animals. $\square$ 